\title{
A Step towards a Field Based Agility Test in Team Sports
}

\author{
David Drake ${ }^{1 *}$, Rodney Kennedy ${ }^{2}$, Jonny Davis ${ }^{1}$, Matthew Godfrey $^{1}$, Simon MacLeod ${ }^{1}$, \\ and Amy Davis ${ }^{1}$
}

\author{
${ }^{1}$ Ulster Rugby, Irish Rugby Football Union (UIster Branch), Northern Ireland \\ ${ }^{2}$ School of Sport, Ulster University, Ireland
}

*Corresponding author: David Drake, Ulster Rugby, Irish Rugby Football Union (Ulster Branch), Kingspan Stadium, 134 Mount Merrion Avenue, Belfast, Northern Ireland E-mail: daviddrake87@gmail.com

\begin{abstract}
Agility performance tests are limited by the requirement for force plates, timing gates or expensive camera systems making application into practice challenging. The primary aim of this study was to assess the inter-rater reliability of a field based $1 \mathrm{v} 1$ agility test encompassing perceptual-action performance. A secondary aim was to assess the relationship between the $1 \mathrm{v} 1$ agility tests with a range of physical performance tests including a commonly used $Y$ step test. The third aim was to contrast the physical performance of high performing players against lower performing players in terms of agility action performance. Twenty-eight male rugby union players volunteered (age $19.3 \pm 2.2$ years, age range $18-24$, body mass $96.5 \pm 13.3 \mathrm{~kg}$ ). Participants were randomly assigned to attack or defensive roles within a simulated rugby evasion task (1v1 agility test). Previously utilized performance scoring (1) was modified to assess agility performance. Two independent investigators reviewed video recordings to score attacking and defensive performance. Cohens Kappa statistic showed inter-rater reliability of agility scoring to be almost perfect, $861(\mathrm{Cl} 0.816$ to 0.917 ). Attacking agility had a large significant relationship with $Y$ step performance $(r=-0.577, p=0.001)$, single leg repeat hop height $(r=0.570, p=0.002)$ and body mass $(r$ $=-0.537, p=0.003)$. Defensive agility outcome had a large significant relationship with $\mathrm{CMJ}$ flight time-contraction time ratio $(r=0.580, p=0.001)$ and $C M J$ concentric duration $(r=$ $-0.656, p=0.000)$. The $Y$ step test shares $33 \%$ of common variance with $1 \mathrm{v} 1$ attacking and $5 \%$ with defensive agility performance likely due to significantly greater frontal and transverse plane movement during agility compared to the change of direction tests. We recommended the $1 \mathrm{v} 1$ agility test be included as part of physical profiling of team sports players.
\end{abstract}

\section{Keywords}

Change of direction, Athletic performance, Perceptual decision making, Rehabilitation, Rugby union

\begin{abstract}
List of Abbreviations
COD: Change of Direction; kg: Kilogram; m: meter; CMJ: Countermovement Jump; SI: Symmetry Index; ES: Effect Size; SD: Standard Deviation; Cl: Confidence Interval; RTP: Return to Play; AFL: Australian Football League; WMA: World Medical Association
\end{abstract}

\section{Introduction}

Agility performance is regarded an essential skill in many team sports including all football codes and court based sports such as basketball, netball and handball [1]. Agility encompasses a perceptual decision-making process and movement action [2] which is achieved by executing a whole-body reorientation strategy [3]. A practical example of agility performance in the context of rugby is when the ball carrier uses deceptive movement to trick a defender into thinking they will run in one direction when they rapidly execute a run in the opposite direction [4]. Performance research has shown evasive agility strategies as key determinants of positive tackle outcomes [5].

Despite advancements in understanding agility performance $[3,4,6]$ and agreement on its importance in sports performance $[1,7]$ implementation of agility tests have been limited in team sports. Agility performance has predominantly been assessing the Change of Direction (COD) movement action in tests such as pro-agility shuttle [8], 505 change of direction test [9], change of direction speed test [10], Y step test [11] and Illinois test [12], that do not involve the assessment of perception [6] and action to a game specific stimulus. The named COD tests principally assess attacking agility actions which further limits their ecological validity in team sports as performance is depen-

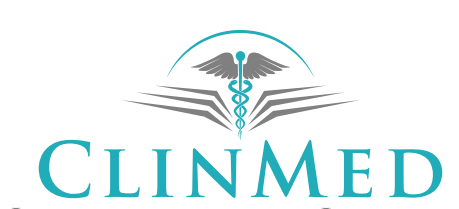

INTERNATIONAL LIBRARY
Citation: Drake D, Kennedy R, Davis J, Godfrey M, MacLeod S, et al. (2017) A Step towards a Field Based Agility Test in Team Sports. Int J Sports Exerc Med 3:079. doi.org/10.23937/2469-5718/1510079 Received: July 31, 2017; Accepted: November 21, 2017; Published: November 23, 2017 Copyright: (c) 2017 Drake D, et al. This is an open-access article distributed under the terms of the Creative Commons Attribution License, which permits unrestricted use, distribution, and reproduction in any medium, provided the original author and source are credited. 
dent upon agility actions in both attacking and defensive scenarios, as has been demonstrated in rugby union [13].

It is recommended during advanced rehabilitation from injury that players should progress from pre-planned change of direction to reactive agility drills with appropriate movement efficiency prior to integrating into team practice activities [14,15]. To enable objective performance criteria for use in athlete-practitioner decision making during the rehabilitation process [14] appropriate agility tests are required. A major hurdle to overcome in implementing agility performance tests with athletes or applying during injury rehabilitation is the need for force plates [16], timing gates [17] and expensive camera systems $[6,17]$. The aims of this study were to assess the reliability and practicality of implementing a field based agility test encompassing perceptual-agility action performance. Secondly, to assess the inter-relationships between the agility test and various athletic performance tests. Lastly, to contrast the physical performance of high performing players against lower performing players in terms of agility action performance.

\section{Methods}

Twenty-eight male rugby union players volunteered to participate in this study (Mean \pm SD age $19.3 \pm 2.2$ years, age range $18-24$, body mass $96.5 \pm 13.3 \mathrm{~kg}$ ). At the time of testing all players were selected in an Academy pathway within a Professional Rugby Union Team and participating regularly in competitive games. Participants undertook an information session explaining test protocols utilized within this study as well as a practical familiarization session of the 1v1 Agility test on a similar artificial pitch surface to the testing venue. Players provided written informed consent and were free to withdraw from the study at any stage without penalty. The study was conducted in accordance with the World Medical Association (WMA) Declaration of Helsinki [18].

\section{Test procedures}

A standardized warm up performed prior to testing, involving 5-minutes jogging, 5-minutes dynamic mobility exercises, five accelerations over 30 meters and five change of direction movements over 10 meters undertaken with progressive intensity as determined by participants. Active recovery followed each warm up activity whereby participants walked back to their starting position. Testing for all players took place on an indoor artificial grass surface with participants wearing shorts, rugby t-shirt and appropriate short studded rugby boots. Participants refrained from any physical exercise for 48 hours prior to testing to ensure the absence of acute fatigue.

\section{Tests of physical performance}

Following previously utilized protocols a series of physical performance tests were completed by all participants under the guidance of the lead investigator, these included; 10-meter acceleration [9], Y step test [11], single leg repeat vertical hop test [19], Countermovement Jump (CMJ) \& isometric squatat an external knee angle of ninety degrees of knee flexion (Isometric squat ${ }^{90}$ ) [20]. All tests except for the isometric squat ${ }^{90}$ were performed prior to the 1v1 Agility test. To reduce potential for fatigue all tests had a 2-minute recovery period between each trial with a 15-minute recovery period following each individual test. At least three trials were completed for each physical performance test, with the average of the best two taken forward for statistical analysis.

\section{1v1 Agility test}

Participants were randomly paired into attacking or defensive roles for each 1v1 dual. This specific rugby task as has been previously used to assess deceptive movement in the side step using virtual reality technology [4] and a video prediction task [21]. A $12 \mathrm{~m} \times 12 \mathrm{~m}$ grid was marked using cones every $1 \mathrm{~m}$ to identify the playing area. Attackers were required to carry a rugby ball and started in the middle at one end of the playing area, whilst defenders were positioned in the middle at the opposite end of the playing area. Objectives and instructions used by Young and Murray [1] were adapted and implemented within this study. The objective of the defending players was to prevent the attacker from scoring a try using defensive agility to move to a position to tackle the attacking player by making contact using both hands around the attacker (below shoulder height in accordance with World Rugby Law 10.5). The objective of the attacking player was to evade the defender without being tackled and score a try by grounding the ball on the line which the defender began the dual. Attacking players triggered the $1 \mathrm{v} 1$ dual, by moving from their static start position. Defending players held a static starting positon and reacted to the attacking players first movement. Players were instructed to stay within the playing area. The simulated tackle was detected as a touch on the attacking player and did not involve direct body contact. Objectives and scoring system were explained to participants during the information ses-

Table 1: Agility test outcome criteria.

\begin{tabular}{|c|c|c|}
\hline Score & Defensive outcome & Attacking outcome \\
\hline 3 & $\begin{array}{l}\text { Both arms around torso simultaneously with elbows } \\
\text { flexed }\end{array}$ & No touch of body at all \\
\hline 2 & $\begin{array}{l}\text { Both arms around torso simultaneously with arms } \\
\text { straight (elbows extended) }\end{array}$ & One hand touch on torso \\
\hline 1 & One hand touch on torso & $\begin{array}{l}\text { Both arms around torso simultaneously with arms straight } \\
\text { (elbows extended) }\end{array}$ \\
\hline 0 & No touch of body at all & $\begin{array}{l}\text { Both arms around torso simultaneously with elbows flexed } \\
\text { or went outside area, or did not cross end line, offended }\end{array}$ \\
\hline
\end{tabular}




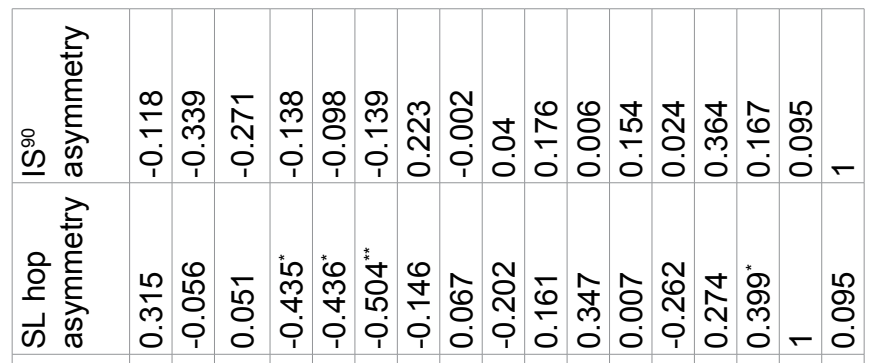

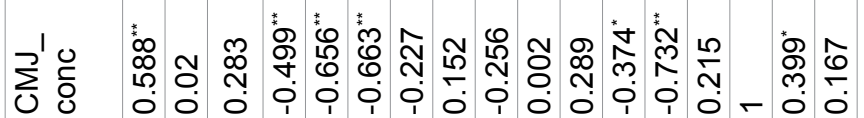

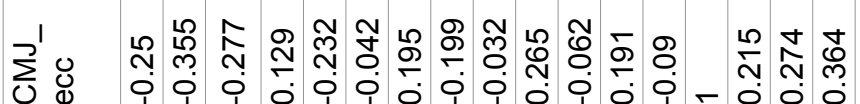

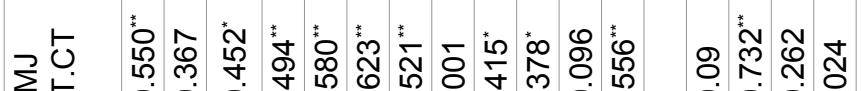
茫

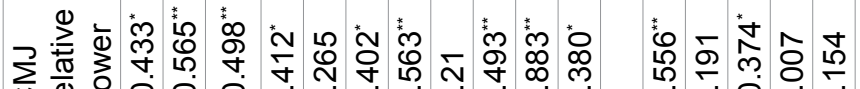

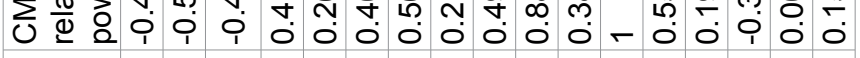

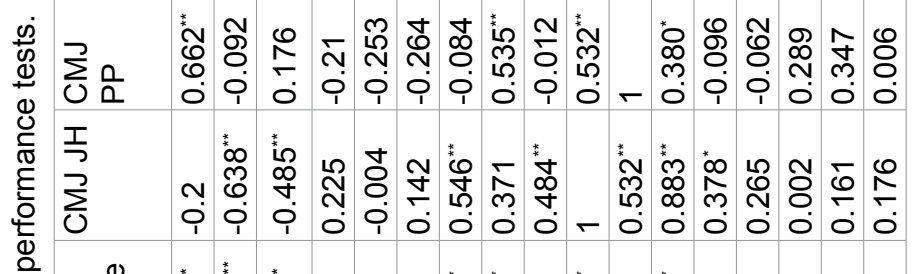

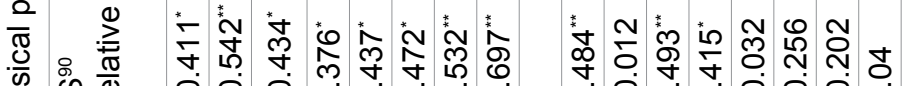

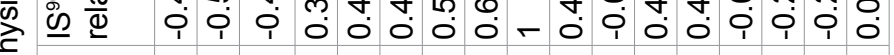
응

ষ্

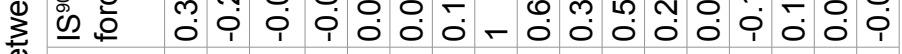
ه 希

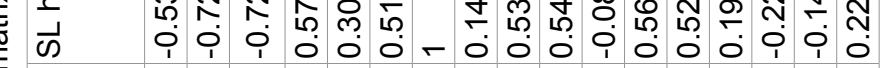

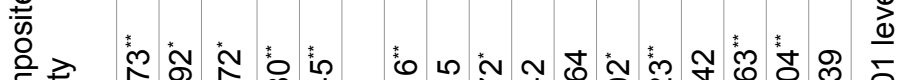

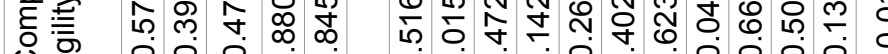

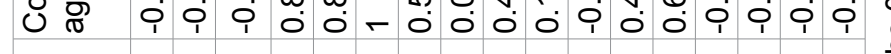

ì

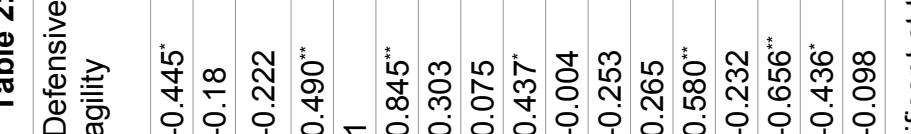

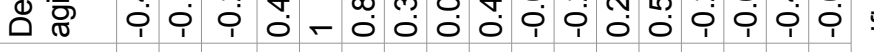

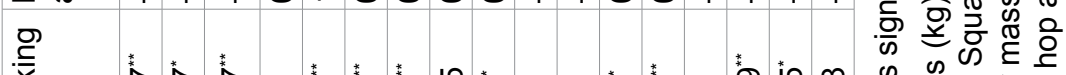

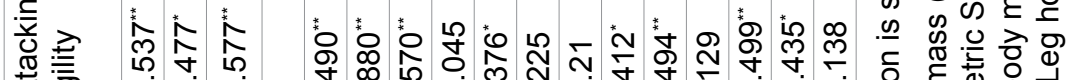
延甭

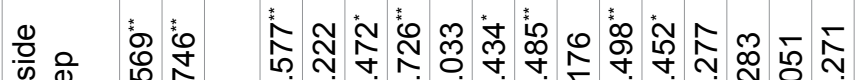
$>$ क

¿ -

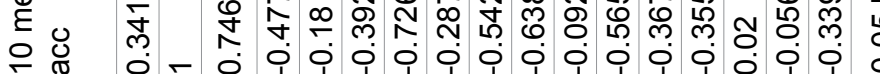

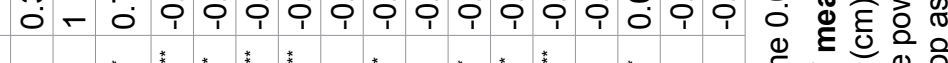

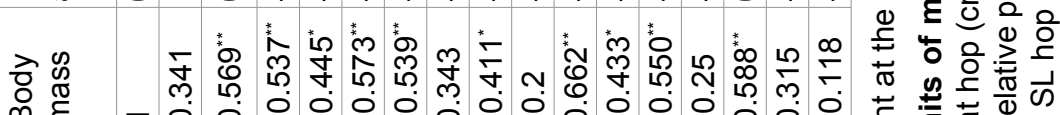

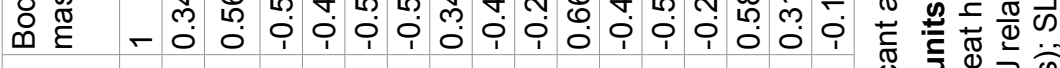

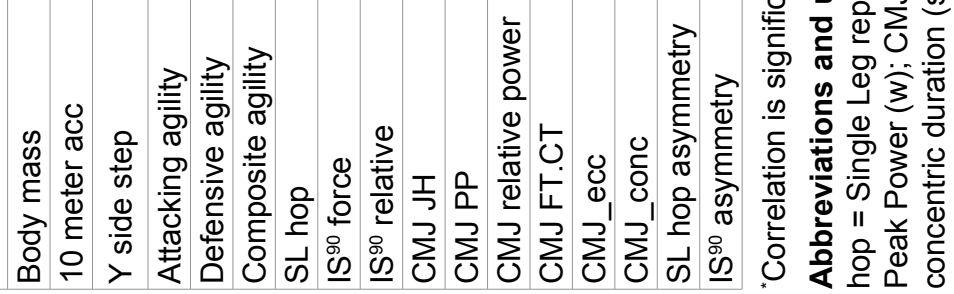


sion and practically demonstrated during the practical familiarization session. Participants were not provided any feedback on their performance in real time. All trials were completed within a 60-minute period, with $1 \mathrm{v} 1$ duals taking place every 30 seconds. Previously utilized agility performance outcome scoring [1] were modified and applied to the coding of agility performance. Descriptive criteria are provided in Table 1 for agility test outcome. Two investigators independently reviewed video footage containing two camera angles (behind the attacker and side on to the playing area) to score the agility test outcome for attacking and defending players. Performance scores for attacking agility and defensive agility were calculated as the total score achieved divided by the best possible score, multiplied by 100 .

\section{Statistical analysis}

Prior to analysis data was visually inspected for normality. A Shapiro-Wilk test assessed the distribution of the data with Levene's test checking the homogeneity of variance. Pearson's correlation coefficient assessed the relationship between agility performance outcome score and physical performance tests, using interpretative thresholds [22]. Statistical significance was set at $p \leq 0.05$. Statistical calculations were performed using IBM SPSS Statistics 22 software (SPSS Inc., Chicago, IL, USA). Kappa (k) statistic was used to assess the level of agreement between two testers rating of agility performance outcomes. Interpretative magnitudes for the level agreement were based on the recommendations of [23] whereby $<0$ less than chance agreement, 0.01-0.20 slight agreement, 0.21-0.40 fair agreement, 0.41-0.60 moderate agreement, $0.61-0.80$ substantial agreement, 0.81-0.99 almost perfect agreement. Independent $t$ tests were used to assess the difference between high performing and lower performing agility groups, determined by median splitting the sampled participant's agility score in accordance with previous grouping methods $[24,25]$. High performers were determined as players with attacking agility $\geq 33 \%$, defensive agility $\geq 67 \%$ and composite agility score $\geq 50 \%$, with scores below this forming the lower performing agility group. Effect Size (ES) was calculated to determine the magnitude of difference between groups and classified as trivial $(<0.2)$, small (0.2-0.6), moderate (0.6-1.2), large (1.2-2.0), and very large (2.0-4.0) [26]. Asymmetries were calculated using the symmetry angle protocol discussed by Zifchock, et al. [27] with asymmetry values transformed to absolute values for statistical analysis. Isometric squat ${ }^{90}$ was performed as a bilateral test, therefore asymmetry was calculated based on the recommendations of Bishop, et al. [28] whereby the Symmetry Index (SI) equation was utilized ( $\mathrm{SI}=$ [larger value-smaller value]/total value *100). Values close to zero constitute nearly perfect between limb symmetry with larger values demonstrating greater asymmetry.

\section{Results}

The Shapiro-Wilk test showed dependent variables were normally distributed in addition to Levene's homogeneity of variance test finding no significant difference between group variance. Inter-rater reliability of agility outcome scores assessed using the Cohens Kappa statistics was 0.861 ( $\mathrm{Cl} 0.816$ to 0.917$)$ with the strength of agreement classified as almost perfect. Correlations between agility outcome scores and physical performance tests are shown in Table 2. Attacking agility outcome had a moderate significant relationship with defensive

Table 3: Comparison between high and lower performing attackers.

\begin{tabular}{|c|c|c|c|c|c|c|c|c|c|c|c|}
\hline & \multicolumn{2}{|c|}{$\begin{array}{l}\text { High } \\
\text { performing } \\
\text { attackers } \\
(n=15)\end{array}$} & \multicolumn{2}{|c|}{$\begin{array}{l}\text { Low } \\
\text { performing } \\
\text { attackers } \\
(n=13)\end{array}$} & \multirow[t]{2}{*}{ Difference } & \multicolumn{2}{|c|}{$\begin{array}{l}95 \% \text { Confidence } \\
\text { Interval of the } \\
\text { Difference }\end{array}$} & \multirow[t]{2}{*}{$p$ value } & \multirow[t]{2}{*}{ ES } & \multicolumn{2}{|c|}{$\begin{array}{l}95 \% \\
\text { Confidence } \\
\text { Interval of the } \\
\text { ES }\end{array}$} \\
\hline & Mean & SD & Mean & SD & & Lower & Upper & & & Lower & Upper \\
\hline Body mass $(\mathrm{kg})$ & 89.13 & 9.95 & 104.9 & 11.70 & -15.77 & -24.179 & -7.367 & 0.001 & -1.46 & -2.249 & -0.588 \\
\hline 10 meter acceleration (s) & 1.759 & 0.065 & 1.833 & 0.047 & -0.074 & -0.119 & -0.030 & 0.002 & -1.29 & -2.069 & -0.445 \\
\hline Y side step (s) & 1.831 & 0.095 & 1.960 & 0.081 & -0.129 & -0.199 & -0.060 & 0.001 & -1.46 & -2.242 & -0.583 \\
\hline Attacking agility (\%) & 56.87 & 15.42 & 20.31 & 10.40 & 36.56 & 26.17 & 46.95 & 0.000 & 2.74 & 1.642 & 3.678 \\
\hline Defensive agility (\%) & 72.47 & 19.00 & 52.54 & 15.29 & 19.93 & 6.385 & 33.47 & 0.006 & 1.15 & 0.315 & 1.910 \\
\hline Composite agility (\%) & 64.53 & 12.78 & 36.31 & 10.40 & 28.23 & 19.08 & 37.37 & 0.00 & 2.40 & 1.372 & 3.296 \\
\hline SL repeat hop $(\mathrm{cm})$ & 16.86 & 2.51 & 12.11 & 2.086 & 4.745 & 2.933 & 6.556 & 0.000 & 2.04 & 1.075 & 2.887 \\
\hline SL repeat hop asymmetry (\%) & 3.827 & 1.884 & 5.000 & 2.715 & -1.173 & -2.969 & 0.622 & 0.191 & -0.51 & -1.248 & 0.260 \\
\hline Iso squat (Net force, $\mathrm{N}$ ) & 1753 & 282.3 & 1746 & 371.2 & 6.929 & -247.3 & 261.1 & 0.956 & 0.02 & -0.722 & 0.763 \\
\hline Iso squat (Relative force, $\mathrm{N} \cdot \mathrm{kg}^{-1}$ ) & 2.007 & 0.222 & 1.700 & 0.358 & 0.307 & 0.079 & 0.535 & 0.01 & 1.05 & 0.228 & 1.806 \\
\hline Iso squat asymmetry (\%) & 3.607 & 2.752 & 4.577 & 3.705 & -0.970 & -3.484 & 1.543 & 0.435 & -0.30 & -1.039 & 0.455 \\
\hline CMJ jump height (m) & 0.395 & 0.047 & 0.366 & 0.050 & 0.029 & -0.009 & 0.067 & 0.127 & 0.60 & -0.177 & 1.339 \\
\hline CMJ peak power (W) & 5083 & 751.2 & 5424 & 576.7 & -341.1 & -867.9 & 185.7 & 0.195 & -0.50 & -1.243 & 0.264 \\
\hline CMJ relative power $\left(\mathrm{W} \cdot \mathrm{kg}^{-1}\right)$ & 56.97 & 4.85 & 52.00 & 5.480 & 4.962 & 0.952 & 8.973 & 0.017 & 0.96 & 0.153 & 1.718 \\
\hline CMJ FT.CT Ratio & 0.791 & 0.123 & 0.678 & 0.090 & 0.113 & 0.028 & 0.198 & 0.011 & 1.04 & 0.220 & 1.796 \\
\hline CMJ_EccDur (s) & 0.193 & 0.027 & 0.190 & 0.054 & 0.003 & -0.030 & 0.035 & 0.866 & 0.06 & -0.680 & 0.806 \\
\hline CMJ_Conc_Dur (s) & 0.240 & 0.033 & 0.268 & 0.024 & -0.028 & -0.051 & -0.006 & 0.016 & -0.97 & -1.729 & -0.163 \\
\hline
\end{tabular}

Abbreviations: Iso Squat = Isometric squat ${ }^{90}$; $\mathrm{CMJ}=$ Countermovement Jump; FT: CT = ratio of Flight Time to Contraction Time; EccDur = Eccentric Duration; Con Dur = Concentric Duration; $s=$ Seconds; $N=$ Newtons; $w=$ watts. 
agility outcome $(r=0.490, p=0.008), 10$-meter acceleration ( $r=-0.477, p=0.01$ ), isometric squat relative strength $(r=0.376, p=0.049)$, CMJ relative power $(r=$ $0.412, p=0.029)$, CMJ flight time-contraction time ratio $(r=0.494, p=0.008)$, CMJ concentric duration $(r=$ $-0.499, p=0.007$ ), and single leg repeat hop asymmetry $(r=-0.435, p=0.021)$. Attacking agility outcome had a large significant relationship with $Y$ step performance $(r$ $=-0.577, p=0.001)$, single leg repeat hop height $(r=$ $0.570, p=0.002)$ and body mass $(r=-0.537, p=0.003)$. Defensive agility outcome had a moderate significant relationship isometric squat relative strength $(r=0.437$, $p=0.002)$, single leg repeat hop asymmetry $(r=-0.436$, $p=0.002)$ and body mass $(r=-0.445, p=0.018)$. Defensive agility outcome had a large significant relationship with CMJ flight time-contraction time ratio $(r=0.580, p$ $=0.001), \mathrm{CMJ}$ concentric duration $(r=-0.656, p=0.000)$.

High performing attackers had significantly faster 10-meter acceleration ( $p=0.002, E S=-1.29)$, faster $Y$ step time $(p=0.001, E S=-1.46)$, higher single leg repeat hop height $(p=0.000, E S=2.04)$, higher relative isometric squat strength $(p=0.01, E S=1.05)$, higher CMJ relative power $(p=0.017, E S=0.96), C M J$ flight time-contraction time ratio $(p=0.011, E S=1.04)$ and faster CMJ concentric duration compared to lower performing players ( $p=0.016$, ES $=-0.97$ ). High performing defenders had significantly higher relative strength $(p$ $=0.003, \mathrm{ES}=1.23), \mathrm{CMJ}$ flight time-contraction ratio ( $\mathrm{p}$ $=0.033, \mathrm{ES}=0.86$ ) and faster $\mathrm{CMJ}$ concentric duration compared to lower performing players $(p=0.006$, ES $=-1.14$ ). Descriptive data for high and low performing players in attacking and defensive agility are presented in Table 3 and Table 4 respectively.

\section{Discussion}

This study aimed to assess the reliability of a field based $1 \mathrm{v} 1$ agility test. Performance scoring of the $1 \mathrm{v} 1$ agility test was found to have almost perfect inter-rater reliability in accordance with previously implemented scoring systems in male AFL players [1]. Nearly perfect scoring agreement between testers is likely a result of simplistic outcome criteria as well as having video footage from multiple angles to enable retrospective scoring. Real time scoring was not used within this study but may be a consideration for physical therapists and strength and conditioning coaches in practical settings without video access or working under time pressures.

A deterministic model of agility performance has been presented by Hewit, et al. [29] with key components underpinning COD performance identified as; between limb asymmetry, relative strength and relative power. We found higher levels of asymmetry in the repeated hop test had a moderate significant relationship with agility performance (attack $r=-0.435, p=0.021$; defense $r=-0.436, p=0.02$ ) but not 10-meter acceleration $(r=-0.056, p=0.776)$ or $Y$ step performance $(r=0.051, p$ $=0.796$ ). Practically, this highlights that players with less asymmetry performed better in the $1 \mathrm{v} 1$ agility, but COD and sprint tasks were not influenced by player asymmetries. Relative strength $\left(\mathrm{IS}^{90}\right)$ assessed in our study had a moderate significant relationship with attacking and defensive performance but relative power (CMJ) was only related to attacking performance. Comparatively we found similar correlations between strength and power qualities with change of direction performance as shown by Spiteri, et al. [16], although the physical

Table 4: Comparison between high and lower performing defenders.

\begin{tabular}{|c|c|c|c|c|c|c|c|c|c|c|c|}
\hline & \multicolumn{2}{|c|}{$\begin{array}{l}\text { High } \\
\text { performing } \\
\text { defenders } \\
(\mathrm{n}=16)\end{array}$} & \multicolumn{2}{|c|}{$\begin{array}{l}\text { Low } \\
\text { performing } \\
\text { defenders } \\
(\mathrm{n}=12)\end{array}$} & \multirow[t]{2}{*}{ Difference } & \multicolumn{2}{|c|}{$\begin{array}{l}95 \% \text { Confidence } \\
\text { Interval of the } \\
\text { Difference }\end{array}$} & \multirow[t]{2}{*}{$p$ value } & \multirow[t]{2}{*}{ ES } & \multicolumn{2}{|c|}{$\begin{array}{l}95 \% \\
\text { Confidence } \\
\text { Interval of the } \\
\text { ES }\end{array}$} \\
\hline & Mean & SD & Mean & SD & & Lower & Upper & & & Lower & Upper \\
\hline Body mass (kg) & 90.91 & 11.40 & 103.85 & 12.30 & 12.94 & -22.19 & -3.68 & 0.0 & -1.10 & -1.864 & 0.266 \\
\hline 10 meter acceler & 1.780 & 0.068 & 1.811 & 0.066 & -0.031 & -0.084 & 0.022 & 0.241 & -0.457 & -1.201 & 0.313 \\
\hline Y side step (s) & 1.878 & 0.111 & 1.908 & 0.109 & -0. & -0.116 & & & -0.267 & -1.010 & 493 \\
\hline Attacking agility (\%) & 48.75 & 20.95 & 28.08 & 20.06 & 20.67 & 4.51 & 36.82 & 0.0 & 1.00 & 0.184 & .766 \\
\hline Defensive agility (\%) & 77.25 & 10.76 & 44.50 & 11.81 & 32.75 & 23.94 & & & 2.92 & 1.780 & 888 \\
\hline Comp & 62.94 & & & & & & & & 2. & 33 & \\
\hline SL repeat hop $(\mathrm{cm})$ & 15.28 & 3.21 & 13.82 & 3.42 & 1.463 & -1.126 & 4.051 & 0.256 & 0.444 & -0.326 & 1.187 \\
\hline SL repeat hop asymme & 3.913 & 2.470 & 4.983 & 2.098 & -1.071 & -2.892 & 0.750 & 0.238 & -0.462 & -1.206 & .309 \\
\hline Iso squat (Net forc & 1787 & 258.6 & 1 & 395.0 & 86.18 & -167.67 & 340.03 & & 0.266 & -0.493 & 1.010 \\
\hline Iso squat (Relative force, $\mathrm{N} \cdot \mathrm{kg}^{-1}$ ) & 2.013 & 0.228 & 1.667 & 0.342 & 0.346 & 0.125 & 0.567 & 0.003 & 1.23 & 0.381 & 2.002 \\
\hline Iso squat asymmetry (\%) & 3.806 & 2.737 & 2 & 3.8 & -0. & -3.1 & & & -0.180 & -0.925 & 0.575 \\
\hline CMJ jump height (m) & 0.384 & 0.045 & 0.379 & 0.057 & 0.005 & -0.035 & 0.045 & 0.785 & 0.106 & -0.646 & 0.852 \\
\hline CMJ peak power (W) & 5080 & 687.3 & 5456 & 649.9 & -376.3 & -903.5 & 151.0 & 0.154 & -0.560 & -1.306 & 0.218 \\
\hline $\mathrm{CMJ}$ relative power $(\mathrm{W}$ & 56.04 & 5.183 & 52.83 & 5.933 & 3.213 & -1.114 & 7.540 & 0.139 & 0.583 & -0.197 & 1.329 \\
\hline CMJ FT.CT Ratio & 0.781 & 0.120 & 0.683 & 0.103 & 0.098 & 0.009 & 0.187 & 0.033 & 0.86 & 0.056 & 1.616 \\
\hline CMJ_EccDur (s) & 0.191 & 0.026 & 0.193 & 0.057 & -0.002 & -0.035 & 0.031 & 0.905 & -0.046 & -0.793 & 0.704 \\
\hline CMJ_Conc_Dur (s) & 0.239 & 0.031 & 0.271 & 0.024 & -0.032 & -0.054 & -0.010 & 0.006 & -1.14 & -1.908 & -0.303 \\
\hline
\end{tabular}

Abbreviations: Iso Squat $=$ Isometric squat ${ }^{90} ; \mathrm{CMJ}=$ Countermovement Jump; FT:CT = ratio of Flight Time to Contraction Time; EccDur = Eccentric Duration; Con Dur = Concentric Duration; $s=$ seconds; $N=$ Newtons; $w=$ watts. 
qualities showed stronger relationships with the $1 \mathrm{v} 1$ agility test than the reactive agility video task used by Spiteri, et al. [16]. This may be due to the greater ecological validity of the $1 \mathrm{v} 1$ agility test compared to the video based reactive agility test. Agility tasks such as a $1 \mathrm{v} 1$ dual [4] allow for greater expression of movement skills in comparison to pre-planned COD tests such as the $Y$ step or $505\left(180^{\circ} \mathrm{COD}\right)$. Differences in movement patterns and perceptual demands of COD compared to agility tests explain the low shared variance between COD and agility tests [25]. Our findings show the $Y$ step shares $33 \%$ of common variance with attacking agility and $5 \%$ with defensive agility performance. Additionally, within our study straight line speed had $23 \%$ and $3 \%$ shared variance with attacking and defensive agility performance. Previous authors have highlighted that COD action performance requires significantly more frontal and transverse plane movement in comparison to straight line speed (e.g., [17]). Given the $Y$ step test had a small non-significant association with defensive agility, we suggest the $Y$ step test is not generalizable to overall agility performance. The importance of defensive agility in team sports has been shown by Wheeler and Sayers [13], whereby poor defensive positioning was shown in $90 \%$ of unsuccessful tackles in professional rugby union players. As such profiling agility performance in rugby should encompass the defense component of performance in addition to the commonly evaluated attacking actions.

$\mathrm{CMJ}$ variables such as flight time to contraction time ratio, eccentric contraction duration and concentric contraction duration assessed within this study have not previously been compared to change of direction or agility performance. CMJ flight time-contraction time ratio, and concentric duration were all significantly higher in high performing attackers and defenders (refer to Table 3). These novel variables may be useful in evaluating training response in terms of neuromuscular function resulting from agility training and support return to play decision making. Further observations were that player body mass had a large negative effect on COD and agility whilst having a moderate non-significant effect on 10-meter acceleration. A comparable result is reported [8] in a study of 64 American football players with an average body mass of $108 \mathrm{~kg}$. Therefore, sports that require high levels of agility performance must consider the cost and benefits from increased player mass.

Conflicting findings on the association between agility performance and injury risk are reported within the current sport and exercise medicine literature. Less agile and slower players had a 2 or 3.5 -fold increased risk respectively for severe injury [30]. Conversely higher performance level in the AFL planned agility test (COD) was significantly linked to greater risk of hip, groin and thigh injures [31]. Whilst the interplay between agility and injury risk is not clear, long lasting deficits in limb symmetry have been shown post return to play in sports persons [32]. The $1 \mathrm{v} 1$ agility test employed within this study was not specifically used to assess limb symmetry as the authors felt the total number of trials performed may be proportionally biased to one limb. However, the scoring criteria could enable the assessment of limb symmetry as criteria for RTP [33] given enough trials were performed between limbs with baseline values established for participants.

\section{Conclusions}

The $1 \mathrm{v} 1$ agility test offers a practical solution to assess agility performance with almost perfect inter-rater reliability without the need for sophisticated equipment. It is recommended that the $1 \mathrm{v} 1$ agility test be included as part of physical performance testing rather than relying solely on linear or COD based tests to evaluate the complexity of agility action performance.

We found similar commonalities in the physical performance qualities underpinning COD performance, but are the first to show such relationships with agility performance inclusive of perceptual-action performance. As the perceptual component of the $1 \mathrm{v} 1$ agility test was not measured, future work may benefit from investigating the perceptual performance using the $1 \mathrm{v} 1$ agility test. Furthermore, the usefulness of the $1 \mathrm{v} 1$ test as a RTP marker warrants future research.

\section{Ethical Statement}

This study was planned and conducted in accordance with the Declaration of Helsinki 2013 [18]. Participants were provided both verbal and written information about the study prior to commencement. The right to withdraw without providing reasons and without prejudice was emphasized.

\section{Conflicts of Interests}

We report no known conflicts of interest.

\section{Funding}

This study received no sources of funding at any stage of completion.

\section{References}

1. Young WB, Murray MP (2017) Reliability of a Field Test of Defending and Attacking Agility in Australian Football and Relationships to Reactive Strength. J Strength Cond Res 31: 509-516.

2. Brughelli M, Cronin J, Levin G, Chaouachi A (2008) Understanding change of direction ability in sport: a review of resistance training studies. Sports Med 38: 1045-1063.

3. Brault S, Bideau B, Craig C, Kulpa R (2010) Balancing deceit and disguise: How to successfully fool the defender in a 1 vs. 1 situation in rugby. Hum Mov Sci 29: 412-425.

4. Brault S, Bideau B, Kulpa R, Craig CM (2012) Detecting deception in movement: the case of the side-step in rugby. PLoS One 7: e37494.

5. Wheeler KW, Sayers MGL (2011) Rugby Union Contact Skills Alter Evasive Agility Performance during Attacking Ball Car- 
ries. International Journal of Sports Science \& Coaching 6 : 419-432.

6. Correia V, Araújo D, Cummins A, Craig CM (2012) Perceiving and acting upon spaces in a VR rugby task: expertise effects in affordance detection and task achievement. J Sport Exerc Psychol 34: 305-321.

7. Paul DJ, Gabbett TJ, Nassis GP (2016) Agility in Team Sports: Testing, Training and Factors Affecting Performance. Sports Med 46: 421-442.

8. Mann BJ, Ivey PA, Mayhew JL, Schumacher RM, Brechue WF (2016) Relationship between agility tests and short sprints: Reliability and smallest worthwhile difference in National Collegiate Athletic Association Division-I football players. J Strength Cond Res 30: 893-900.

9. Nimphius S, Callaghan SJ, Spiteri T, Lockie RG (2016) Change of Direction Deficit: A More Isolated Measure of Change of Direction Performance Than Total 505 Time. J Strength Cond Res 30: 3024-3032.

10. Sheppard JM, Young WB, Doyle TL, Sheppard TA, Newton RU (2006) An evaluation of a new test of reactive agility and its relationship to sprint speed and change of direction speed. J Sci Med Sport 9: 342-349.

11. Lockie RG, Schultz AB, McGann TS, Jalilvand F, Callaghan SJ (2015) Peak ankle muscle activity of faster and slower basketball players during the change of direction step in a reactive cutting task. J Athlet Enhancement 6: 17-20.

12. Hachana $Y$, Chaabène $H$, Nabli MA, Attia A, Moualhi J, et al. (2013) Test-Retest Reliability, Criterion-Related Validity, and Minimal Detectable Change of the Illinois Agility Test in Male Team Sport Athletes. J Strength Cond Res 27: 2752-2759.

13. Wheeler K, Sayers M (2009) Contact skills predicting tackle-breaks in Rugby Union. International Journal of Sports Science \& Coaching 4: 535-544.

14. Sclafani MP, Davis CC (2016) Return to play progression for rugby following injury to the lower extremity: a clinical commentary and review of the literature. Int $\mathrm{J}$ Sports Phys Ther 11: 302-320.

15. Reiman MP, Lorenz DS (2011) Integration of strength and conditioning principles into a rehabilitation program. Int $J$ Sports Phys Ther 6: 241-253.

16. Spiteri T, Nimphius S, Hart NH, Specos C, Sheppard JM, et al. (2014) Contribution of strength characteristics to change of direction and agility performance in female basketball athletes. J Strength Cond Res 28: 2415-2423.

17. Green BS, Blake C, Caulfield BM (2011) A comparison of cutting technique performance in rugby union players. $J$ Strength Cond Res 25: 2668-2680.

18. WMA (2013) World Medical Association Declaration of Helsinki: Ethical principles for medical research involving human subjects. JAMA 310: 2191-2194.
19. Maulder P, Cronin J (2005) Horizontal and vertical jump assessment: Reliability, symmetry, discriminative and predictive ability. Physical Therapy in Sport 6: 74-82.

20. Kennedy RA, Drake D (2017) Dissociated time course of recovery between strength and power after isoinertial resistance loading in rugby union players. J Strength Cond Res.

21. Jackson RC, Warren S, Abernethy B (2006) Anticipation skill and susceptibility to deceptive movement. Acta Psychol (Amst) 123: 355-371.

22. Hopkins WG (2002) A new view of statistics.

23. Viera AJ, Garrett JM (2005) Understanding interobserver agreement: The kappa statistic. Fam Med 37: 360-363.

24. Maloney SJ, Richards J, Nixon DG, Harvey LJ, Fletcher IM (2017) Do stiffness and asymmetries predict change of direction performance? J Sports Sci 35: 547-556.

25. Young WB, Miller IR, Talpey SW (2015) Physical qualities predict change-of-direction speed but not defensive agility in Australian rules football. J Strength Cond Res 29: 206212.

26. Hopkins WG, Marshall SW, Batterham AM, Hanin J (2009) Progressive statistics for studies in sports medicine and exercise science. Med Sci Sports Exerc 41: 3-13.

27. Zifchock RA, Davis I, Higginson J, Royer T (2008) The symmetry angle: a novel, robust method of quantifying asymmetry. Gait Posture 27: 622-627.

28. Bishop C, Read P, Chavda S, Turner A (2016) A symmetries of the lower limb: The calculation conundrum in strength training and conditioning. Strength \& Conditioning Journal 38: 27-32.

29. Hewit JK, Cronin JB, Hume PA (2012) Understanding change of direction performance: a technical analysis of a 180 aerial catch and turn task. International Journal of Sports Science \& Coaching 7: 503-514

30. Mirsafaei Rizi R, Yeung SS, Stewart NJ, Yeung EW (2017) Risk factors that predict severe injuries in university rugby sevens players. J Sci Med Sport 20: 648-652.

31. Chalmers S, Magarey ME, Esterman A, Speechley M, Scase E, et al. (2013) The relationship between pre-season fitness testing and injury in elite junior Australian football players. J Sci Med Sport 16: 307-311.

32. Myer GD, Martin L, Ford KR, Paterno MV, Schmitt LC, et al. (2012) No association of time from surgery with functional deficits in athletes after anterior cruciate ligament reconstruction: evidence for objective return-to-sport criteria. Am J Sports Med 40: 2256-2263.

33. Myer GD, Paterno MV, Ford KR, Quatman CE, Hewett TE (2006) Rehabilitation after anterior cruciate ligament reconstruction: criteria-based progression through the return-tosport phase. J Orthop Sports Phys Ther 36: 385-402. 